\title{
Stromal "activation" markers do not confer pathogenic activity in tendinopathy
}

\author{
Lindsay A. N. Crowe ${ }^{1}$ | Emma Garcia Melchor ${ }^{1}$ | George A. C. Murrell ${ }^{2}$ | Iain \\ B. McInnes ${ }^{1}$ | Moeed Akbar ${ }^{1}$ | Neal L. Millar ${ }^{1}$ (D)
}

${ }^{1}$ Institute of Infection, Immunity and Inflammation, College of Medicine, Veterinary and Life Sciences, University of Glasgow, Glasgow, UK

${ }^{2}$ Orthopaedic Research Institute, St George Hospital Campus, University of New South Wales, Sydney, NSW, Australia

\section{Correspondence}

Neal L. Millar, Institute of Infection, Immunity and Inflammation, College of Medicine, Veterinary and Life Sciences University of Glasgow, 120 University Avenue Glasgow G12 8TA, UK.

Email: neal.millar@glasgow.ac.uk

Funding informationThis work was funded by the Medical Research Council (MR/R020515/1).
Tendinopathy is a highly prevalent musculoskeletal pathology associated with incremental injury as result of repetitive microtrauma. We sought to explore the physiological significance of stromal "activation" signatures in a human model of tendinopathy. Torn supraspinatus tendon and matched intact subscapularis tendon biopsies were collected from patients undergoing shoulder surgery while healthy tendon was collected from patients undergoing anterior cruciate ligament (ACL) reconstruction. Expression of stromal activation markers was analyzed at transcript/ protein level using qRT-PCR and immunohistochemistry. Gene expression of stromal activation markers was silenced by siRNA-mediated knockdown or induced by IL-1 $\beta$ stimulation. Expression of "activation" markers podoplanin, VCAM-1 and CD248 was identified in human tendon tissue. Podoplanin and VCAM-1 expression were significantly increased in tendinopathic tissue. Knockdown of podoplanin and VCAM-1 in normal and tendinopathic tenocytes did not have any significant effect on expression of matrix genes COL1A1, COL3A1, TNC, or DCN. Similarly, no changes in release of inflammatory mediators IL-6, IL-8, and CCL2 were observed in podoplanin/VCAM-1 knockdown cultures. Our data suggest that silencing expression of stromal "activation" markers does not affect the intrinsic inflammatory profile or matrix regulatory behavior of tenocytes. We propose that the term "activation" is more appropriately reflected by alterations in tenocyte behavior that induce changes in the stromal microenvironment and overall tissue architecture rather than identification through potentially arbitrary phenotypic traits.

\section{K E Y W O R D S}

inflammation, stroma, T cells, tendinopathy, tenocytes

\section{$1 \mid$ INTRODUCTION}

Tendinopathy is a highly prevalent musculoskeletal pathology associated with overuse injury. Tendinopathies account for $30 \%-50 \%$ of sporting injuries and a large portion of orthopedic referrals from primary care. ${ }^{1,2}$ Dysregulated matrix repair elicited by persistent pathological inflammation is the main causative factor in the development of established tendinopathy. ${ }^{3}$ Injured tendons display irregular tissue architecture and weakened biomechanical properties, primarily

This is an open access article under the terms of the Creative Commons Attribution License, which permits use, distribution and reproduction in any medium, provided the original 
attributed to altered collagen synthesis and matrix deposition. ${ }^{4}$ Furthermore, an incomplete healing response is common and consequent propensity for re-injury presents many challenges in a clinical setting. ${ }^{5}$

Historically, the stroma was considered to be immunologically inert and exist purely as a tissue-specific scaffold that participates in wound healing responses. ${ }^{6}$ Excessive loading of tendons was considered the main pathological stimulus for degeneration, conferred by the mechanosensing properties of tenocytes. ${ }^{7}$ Tenocytes are fibroblast-like cells that comprise the main cellular component of the tendon stroma. Tenocytes regulate the structure and function of healthy tendons and participate in tissue repair following periods of acute inflammation. ${ }^{5}$ It has been postulated that recruitment, influx, and retention of immune cells are controlled by cytokine and chemokine gradients created by resident stromal cells. ${ }^{8}$

It is thought stromal cell properties are defined according to tissue structure at an individual site and the external stimuli they receive. ${ }^{9}$ For example, tenocytes are interspersed uniformly throughout the tendon matrix and are adapted to respond to mechanical force and damage-associated molecular patterns (DAMPs) through pattern recognition receptors. ${ }^{10}$ Recent studies have suggested that repeated exposure to pathological stimuli induce epigenetic changes that drive a persistent state of activation. ${ }^{11,12}$ Under certain conditions, such as chronic inflammation, it has been postulated that these genetic changes are accompanied by phenotypic alterations including constitutive expression of cell surface receptors termed stromal "activation" markers. ${ }^{13}$ Traditionally, fibroblasts were perceived as a homogenous population with a relatively limited functional capacity. However, emerging evidence suggests that fibroblasts display a degree of plasticity and can exist as functionally distinct populations analogous to subsets of leukocytes. ${ }^{14}$

Studies have highlighted the presence of "activation" markers in several inflammatory pathologies including rheumatoid arthritis (RA) and cancer where surface marker expression has been associated with "aggressive" and "invasive" stromal cell phenotypes. ${ }^{15-17}$ More recently, their presence has been documented in soft tissue diseases including adhesive capsulitis and tendinopathy; however, evidence pertaining to their functional significance in the context of inflammation and matrix regulation is limited. ${ }^{18,19}$

A recent study reported sustained expression of stromal fibroblast activation markers podoplanin, vascular cell adhesion molecule 1 (VCAM-1/CD106), and CD248 (endosialin/ tumor endothelial marker-1) in tendinopathic biopsy samples that persisted up to 4 years post-treatment. It was also reported this sustained stromal activation marker expression was induced in cultured cells in response to an inflammatory stimulus. ${ }^{19}$ Podoplanin is a small cell surface mucin-like glycoprotein expressed on a number of cells including fibroblasts and macrophages. Its expression is upregulated under high levels of inflammation associated with chronic conditions such as RA, psoriasis, and multiple sclerosis (MS). ${ }^{20}$ :Little is known about the physiological properties of podoplanin; however, recent studies have highlighted its role in regulating the inflammatory reaction and immune cell infiltration during sepsis. ${ }^{21}$ VCAM-1 (vascular cell adhesion molecule-1) expression is associated with the progression of several immunological disorders including RA, asthma, and cancer. ${ }^{22}$ It is primarily expressed on the surface of endothelial cells; however, its expression is upregulated in other cell types including fibroblasts and macrophages under inflammatory conditions. ${ }^{23} \mathrm{CD} 248$ is a transmembrane glycoprotein highly expressed in most cancers, RA, and diseases associated with excessive fibrosis. ${ }^{24}$ In addition, CD248 acts as a receptor for extracellular matrix proteins including collagen I and fibronectin. ${ }^{25}$

The immune landscape in tendinopathy is diverse and host to several functionally and phenotypically distinct populations of cells. "Activation" proteins are generally absent from the stroma of normal tissue which suggests they play a role in disease pathology. ${ }^{26}$ This study sought to identify relationships between expression of surface "activation" proteins and regulation of inflammation and matrix turnover by tenocytes using a loss-of-function approach. The ultimate aim of this work was to gain insight into the role of these proteins within the tendon stroma with regard to inflammation and ECM turnover and how their expression may relate to persistence of inflammation in tendinopathy.

\section{2 | MATERIALS AND METHODS}

\section{1 | Human model of tendinopathy}

All procedures and protocols were approved by the Ethics Committee under approval numbers Central Network, South East Health (HREC/96/55, HREC/14/130) and West of Scotland REC (REC14/WS/1035) with informed consent obtained and carried out in accordance with standard operative procedures. For immunohistochemistry, ten supraspinatus tendon samples were collected from patients with rotator cuff tears undergoing shoulder surgery. The mean age of the rotator cuff ruptured patients was 50 years (range, 30-62 years) - the mean tear size was $2.2 \mathrm{~cm}^{2}$ (range $1-4 \mathrm{~cm}^{2}$ ). Samples of the subscapularis tendon were also collected from the same patients. Patients were only included if there was no clinically detectable evidence of subscapularis tendinopathy on a preoperative MRI scan as determined by a musculoskeletal radiologist 
or macroscopic damage to the subscapularis tendon at the time of arthroscopy as determined by the senior author (NLM) - by these criteria, they represented a preclinical cohort. In this cohort, all patients fulfilled the following criteria: (a) a history of shoulder pain and dysfunction, (b) no previous surgery on the affected shoulder, (c) no radiographic sign of fracture of the shoulder, and (d) no history of RA or osteoarthritis.

An independent control group was obtained comprising ten samples of subscapularis tendon collected from patients undergoing arthroscopic surgery for shoulder stabilization without rotator cuff tears, no previous shoulder surgery, no radiographic signs of shoulder fracture, or history of RA or OA. The absence of rotator cuff tears was confirmed by arthroscopic examination. The mean age of the control group was 23 years (range, 16-28 years). For mRNA expression, fourteen (mean 49 years range, 30-65 years) early tendinopathy (subscapularis) samples, 12 late (mean 54 years range, 38-68 years, mean tear size $2.0 \mathrm{~cm}^{2}$ (range $1-4.5 \mathrm{~cm}^{2}$ ).) tendinopathy (supraspinatus) samples were utilized while 8 hamstring tendons (mean 23 years (range, 15-25 years) obtained at the time of routine anterior cruciate ligament reconstruction were employed as an independent control group. Additionally, standardized patient demographics were obtained preoperatively and included the duration of shoulder symptoms experienced by the patient and the number of subacromial steroid injections.

\section{2 $\quad$ Cell culture and treatments}

Normal and tendinopathic human tendon-derived cells were explanted form hamstring tendons of patients undergoing anterior ligament reconstruction or supraspinatus tendon from patients undergoing rotator cuff repair, respectively. Patients were age ranged from 18 to 25 . Hamstring tendons were cut into $2 \mathrm{~mm}^{3}$ pieces and placed in RPMI 1640 supplemented with $10 \%$ heat-inactivated fetal bovine serum (FBS), $100 \mu \mathrm{g} / \mathrm{mL}$ penicillin, $100 \mu \mathrm{g} / \mathrm{mL}$ streptomycin, and $2 \mathrm{nmol} / \mathrm{L}$ L-glutamine (all Thermo Fisher Scientific). Explants were maintained in a humidified environment at $37^{\circ} \mathrm{C}, 5 \% \mathrm{CO}_{2}$ to allow tenocytes to adhere. Medium was replenished every 5 days until cells were confluent. Tissue was then removed, and cells were placed in fresh medium. Cells were passaged using trypsin-EDTA (Sigma-Aldrich). Normal and tendinopathic tenocytes from the second or third passage were seeded at $2.5 \times 10^{4} / \mathrm{mL}$ in 24 well culture plates and allowed to rest for 48 hours prior to siRNA transfection and stimulation.

\section{3 | siRNA transfection and tenocyte stimulation}

In order to knockdown proteins expressed on the tenocyte surface, tenocytes were transfected using Silencer Select predesigned siRNAs (PDPN, assay ID S20884, VCAM-1 assay ID S14760, Thermo Fisher Scientific) or a scrambled negative control (miRIDIAN microRNA Mimic Negative Control \#1, Horizon). The following transfection protocol using DharmaFECT 3 (Dharmacon) was adapted from the manufacturer's recommended protocol. DharmaFECT 3 and OptiMEM (Thermo Fisher Scientific) were added to an eppendorf. $10 \mu \mathrm{mol} / \mathrm{L}$ stock concentration siRNA and OptiMEM were added to a separate eppendorf and incubated at room temperature for five minutes. The contents of both were subsequently mixed and incubated at room temperature for 15 minutes. The medium in which the cells were cultured was replenished with $200 \mu \mathrm{L}$ of fresh medium and $50 \mu \mathrm{L}$ of the transfection mixture was added for a total volume of $250 \mu \mathrm{L}$ ( siRNA concentration $25 \mathrm{nmol} / \mathrm{L}$ ) in a 24 well plate. The cells were incubated at $37^{\circ} \mathrm{C}$ in a $\mathrm{CO}_{2}$ incubator for 48 hours. Cells were then retransfected for a further 24 hours using the same protocol. Transcript and protein knockdown were measured by qPCR and flow cytometry on a BD Fortessa, respectively. Antibodies for flow cytometry were purchased from Biolegend (Table 1).

Following 72 hours transfection, cells were washed and medium was replaced with RPMI containing $10 \mathrm{ng} / \mathrm{mL}$ recombinant human IL-1 $\beta$ and incubated for 24 hours before harvesting of supernatants and cell lysates.

\subsection{RNA extraction, cDNA synthesis, and real-time qPCR}

Tendon tissue was placed in RNA later solution (Ambion) and stored at $-20^{\circ} \mathrm{C}$. Tissue samples were homogenized in PureLink lysis buffer containing 1\% 2-mercaptoethanol using a Qiagen Tissue Lyser LT. Total RNA was isolated using the PureLink RNA Mini Kit (Thermo Fisher Scientific) according to manufacturer's instructions.

\begin{tabular}{|c|c|c|c|c|}
\hline & Fluorophore & Clone & Isotype & $\begin{array}{l}\text { Catalogue } \\
\text { number }\end{array}$ \\
\hline Podoplanin & PerCP/Cy5.5 & NC-08 & Rat IgG2a k & 337011 \\
\hline VCAM-1 & $\mathrm{PE}$ & STA & Mouse IgG1 k & 305805 \\
\hline
\end{tabular}

TA B L E 1 Antibodies used for flow cytometry 
TA B L E 2 Primer sequences

\begin{tabular}{|c|c|c|}
\hline Target & Forward & Reverse \\
\hline Col1a1 & 5'-CAATGCTGCCCTTTCTGCTCC-3' & 5'-CACTTGGGTGTTTGAGCATTG-3' \\
\hline Col3a1 & 5'- TATCGAACACGCAAGGCTGTG-3' & 5'-CACTTGGGTGTTTGAGCATTG-3' \\
\hline Tenascin C & 5'-CTTTGGCTGGGTTGCTTGAC-3' & 5'-GTGCCAGGAGACCGTACCAC-3' \\
\hline Decorin & 5'-CGCCTCATCTGAGGGAGCTT-3' & 5'-TACTGGACCGGGTTGCTGAA-3' \\
\hline VCAM1 & 5'-GCAAGTCTACATATCACCCAAGA-3' & 5'-TAGACCCTGGCTGGAACA-3' \\
\hline $\mathrm{CD} 248$ & 5'-CTGTGCTCGGCAAGACC-3' & 5'-CCCAAATCCCAAGGGAAGAT-3' \\
\hline
\end{tabular}

Cells were placed in PureLink lysis buffer containing 1\% 2-mercaptoethanol, and RNA was extracted using mini columns according to the PureLink protocol. RNA concentration and purity were determined using a spectrophotometer (Nanodrop 2000, Thermo Scientific). 100 ng of RNA was converted to cDNA using High Capacity cDNA reverse transcription kit (Thermo Fisher Scientific) according to manufacturer's instructions. cDNA was diluted 1 in 5 using RNase free water. qPCR was performed using PowerUp Sybr Green Mastermix (Thermo Fisher Scientific) and $1 \mu \mathrm{l}$ cDNA was used per reaction with $0.1 \mu \mathrm{mol} / \mathrm{L}$ of forward and reverse primers. Each sample was run in duplicate and normalized to GAPDH or $18 \mathrm{~S}$ endogenous control. Data represent relative mRNA expression $\left(2^{-\Delta \mathrm{CT}}\right)$ or fold change from untreated cells $\left(2^{-\Delta \Delta \mathrm{CT}}\right)$ (Table 2$)$.

Primers (Integrated DNA Technologies) were as follows:

\section{5 | Measurement of cytokine release by ELISA}

Cell culture supernatants were collected from transfected tenocytes and the concentrations of IL-6, IL-8, and CCL2 were determined using commercially available ELISA kits (Thermo Fisher Scientific). Cell culture supernatants were diluted with assay diluent to achieve concentrations within the specified range. Optical density was measured at $450 \mathrm{~nm}$ by a microplate reader.

\subsection{Histology and immunohistochemistry}

Samples were stained with H\&E and toluidine blue for determination of the degree of tendinopathy as assessed by a modified version of the Bonar score (Grade $4=$ marked tendinopathy, Grade 3 = advanced tendinopathy, Grade $2=$ moderate degeneration, Grade $1=$ mild degeneration, Grade $0=$ normal tendon). This included the presence or absence of edema and degeneration together with the degree of fibroblast cellularity and chondroid metaplasia. Thereafter, sections were stained with a range of primary antibodies directed against the following markers: podoplanin (Biolegend, Cambridge UK), VCAM-1, CD248 (R and D systems, UK).

Endogenous peroxidase activity was quenched with $3 \%$ (v/v) $\mathrm{H}_{2} \mathrm{O}_{2}$, and non-specific antibody binding blocked with $2.5 \%$ horse serum in Tris buffered saline (TBS) solution with detergent Tween20 (TBST) buffer for 30 minutes. Antigen retrieval was performed in $0.01 \mathrm{M}$ citrate buffer for $20 \mathrm{~min}$ utes in a microwave. Sections were incubated with primary antibody in $2.5 \%(\mathrm{w} / \mathrm{v})$ horse serum/human serum/TBST at $4^{\circ} \mathrm{C}$ overnight. After two washes, slides were incubated with Vector ImmPRESS Reagent kit as per manufacturer's instructions for 30 minutes. The slides were washed and incubated with Vector ImmPACT DAB chromagen solution for 2 minutes followed by extensive washing. Finally, the sections were counterstained with hematoxylin.

Images were captured using Apple Open laboratory software. Positive (human tonsil tissue) control specimens were included, in addition to the surgical specimens for each individual antibody staining technique. Omission of primary antibody and use of negative control (human OA samples and human tendon samples) isotypes and a positive control (human tonsil samples) confirmed the specificity of staining.

We applied a scoring system based on previous methods to quantify the immunohistochemical staining. Five random high-power fields ( $\times 400$ magnification) were evaluated by two independent assessors (NLM,GACM). In each field, the number of positive and negatively stained cells was counted and the percentage of positive cells was calculated.

\section{$2.7 \quad$ Statistical analysis}

Comparisons between groups were made with KruskalWallis One-Way Analysis of Variance on ranks, Ordinary One-Way ANOVA with Tukey's correction for multiple comparisons, Friedman test with Dunn's correction for multiple comparisons, using Graph Pad Prism 5 software. In all analyses, $P<.05$ was considered statistically significant. 


\section{3 | RESULTS}

\subsection{Podoplanin and VCAM-1 expression is increased in tendinopathic tissue}

RT-PCR of whole tendon tissue showed a significant increase in podoplanin and VCAM-1 expression between control and late tendinopathy (Figure 1A,B $P<.01$ ). A modest increase in expression of both markers was observed in early tendinopathy; however, this was not significant vs control or late tendinopathic samples. No apparent changes in expression of CD248 transcript were observed (Figure 1C). Immunohistochemical staining of tendinopathic sections showed positive staining of PDPN, VCAM-1, and CD248 in both control and tendinopathic sections (Figure 1D-H). Quantitative analysis revealed significant increases in podoplanin $(P<.05$ control vs late tendinopathy) and VCAM-1 $(P<.05$ control vs late tendinopathy) expression between control and tendinopathic sections (Figure 1E-I). Conversely, CD248 expression remained constant between control and both disease groups.

We next examined the effect of an inflammatory stimulus on expression of PDPN, VCAM-1, and CD248 which showed that the inflammatory molecules IL- $1 \beta$ and TNF- $\alpha$ increased expression of $P D P N$, approximately 2.5 fold in both instances (Figure S1A). TNF- $\alpha$ induced a significant increase in $V C A M-1$ expression (Figure $\mathrm{S} 1 \mathrm{~B}, P<.05$ ). IL- $1 \beta$ stimulation also resulted in a marked increase in VCAM-1 expression. Interestingly, upon stimulation, expression of $C D 248$ in tenocytes appeared to decrease slightly (Figure S1C).

\section{2 | Podoplanin and VCAM-1 knockdown have no direct effect on the inflammatory secretome of tenocytes}

In order to assess the effect of the presence and relative depletion of podoplanin and VCAM-1 expression on the intrinsic behavior of tenocytes, we developed an siRNA knockdown procedure using commercially available siRNA. On average, we obtained $80 \%$ knockdown in podoplanin protein expression and $92 \%$ reduction in PDPN transcript by RTPCR (Figure S2). Similarly, we observed an average reduction of 71\% VCAM-1 protein and 90\% VCAM-1 transcript in siRNA-transfected tenocytes relative to scramble control (Figure S2).

To test the effect of stromal surface marker knockdown on the inflammatory secretome, normal and tendinopathic tenocytes were subject to podoplanin and VCAM-1 knockdown and subsequently stimulated with $10 \mathrm{ng} / \mathrm{mL}$ IL- $1 \beta$ to invoke an inflammatory response. Addition of IL- $1 \beta$ to the culture system induced significant increases in release of IL-6, IL-8, and CCL2 from tenocytes compared with controls (untransfected control, scramble control and podoplanin/ VCAM-1 siRNA vs IL-1 $\beta$ stimulation) (Figure $2, P<.05$, 0.01 or 0.001 ) (Figure 3). We found no significant effect of podoplanin or VCAM1 knockdown alone on release of IL-6, IL-8 (Figure 4C,D, Figure 5C,D), or CCL2 (Figure 4E,F, Figure 5E,F) in normal or tendinopathic tenocytes compared with untransfected tenocytes and scramble transfection control. Similarly, knockdown of podoplanin or VCAM-1 did not affect the magnitude of cytokine/chemokine release in response to IL-1 $\beta$ stimulation.

\section{3 | Podoplanin or VCAM-1 knockdown does not alter expression of matrix proteins in tenocytes}

The effect of podoplanin and VCAM-1 knockdown and simultaneous stimulation with IL- $1 \beta$ on matrix production was measured by RT-qPCR using a panel of genes associated with matrix regulation by tenocytes. Neither podoplanin knockdown nor IL-1 $\beta$ stimulation appeared to have any effect on production of collagen 1 (COLIAI) in normal and tendinopathic tenocytes (Figure 6A,B). There was a trend toward increased expression of matrix proteins associated with tendinopathy including collagen 3 (COL3AI) (Figure 6C,D), tenascin $\mathrm{C}(T N C)$ (Figure 6E,F), and decorin $(D C N)$ (Figure $6 \mathrm{G}, \mathrm{H}$ ) in response to IL- $1 \beta$ stimulation in normal and tendinopathic tenocytes; however, podoplanin knockdown did not appear to have any demonstrable effect.

Similarly, VCAM-1 knockdown did not appear to have any effect on expression on matrix proteins in normal or tendinopathic tenocytes compared with control (Figure 7A-J). As observed previously, there was a trend of increased expression of COL3Al (Figure 7C,D) and TNC (Figure 7G,H) in normal and tendinopathic tenocytes in response to IL-1 $\beta$ stimulation.

\section{4 | DISCUSSION}

In the context of the tendinopathy, little is known about the properties of stromal cells and whether phenotypic characteristics may direct pathology. This study sought to address the physiological significance of stromal "activation" markers in tendinopathy using an in vitro loss-of-function approach. Data from our human tendinopathy model showed significant variations in podoplanin and VCAM-1 expression between healthy and diseased states which is in agreement with previous findings. ${ }^{19}$ We did not, however, observe any changes in CD248 between control and tendinopathy groups as anticipated in any of our ex vivo or in vitro studies. Consequently, we proceeded to knock down expression of podoplanin and VCAM-1 in normal and tendinopathic tenocytes. 
(A)

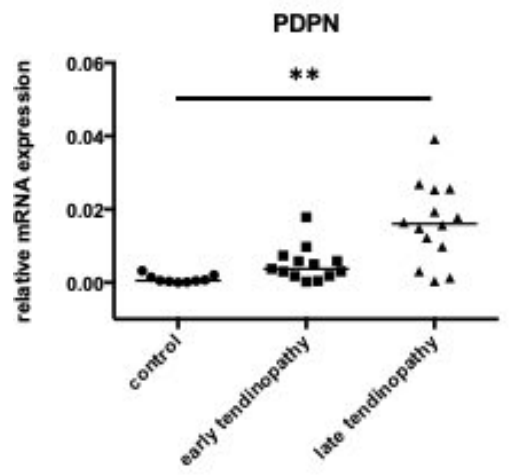

(B)

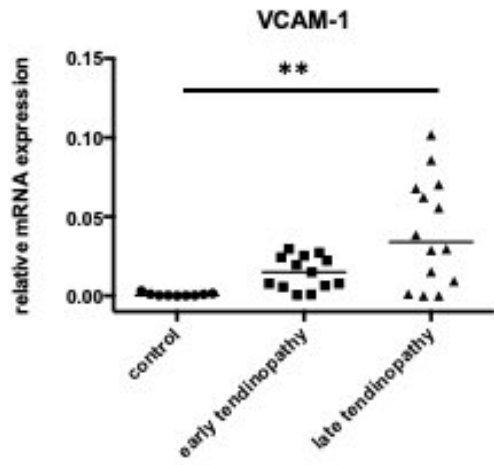

PDPN Tendinopathy

(D)

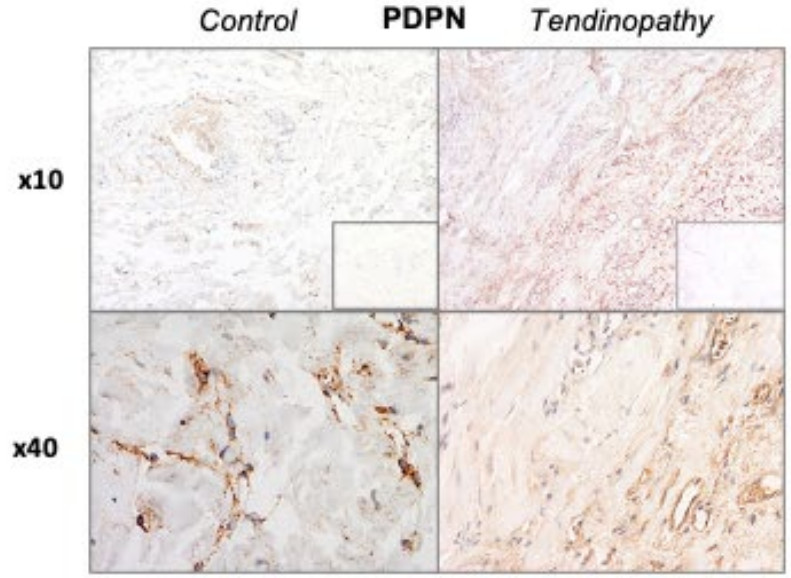

(F)

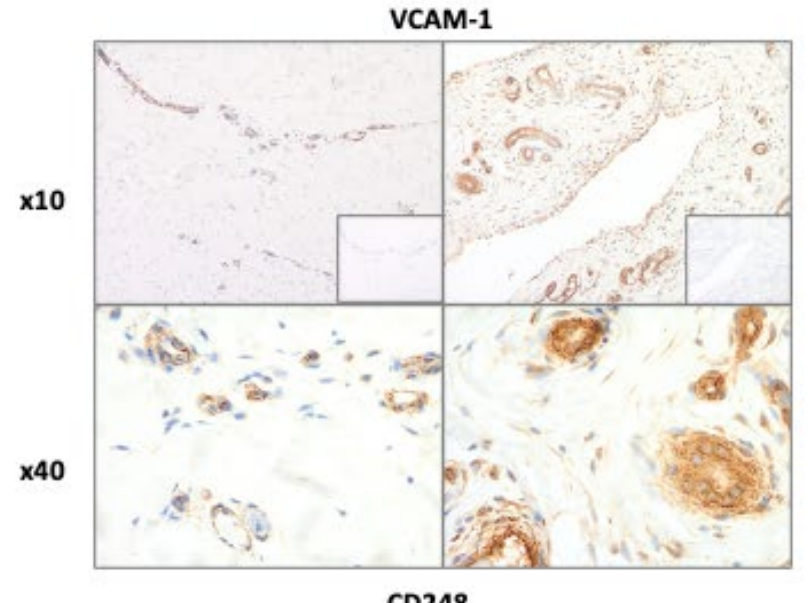

(H)

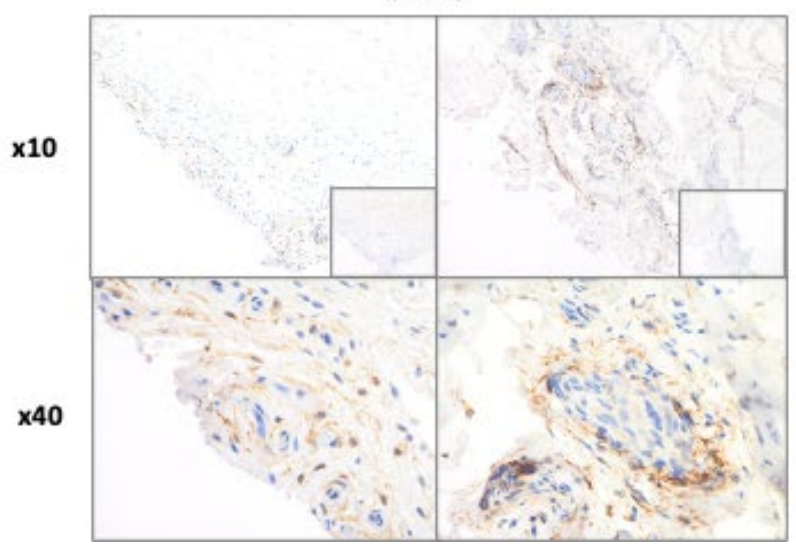

(C)

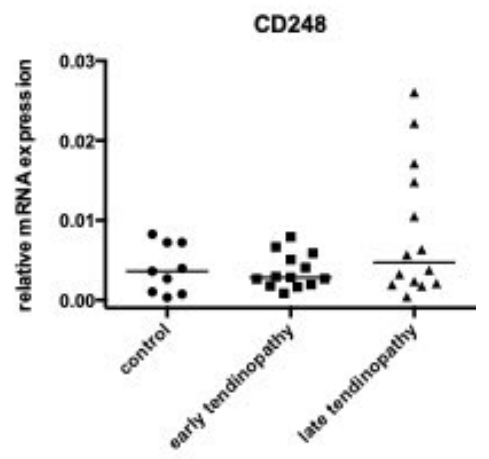

(E)

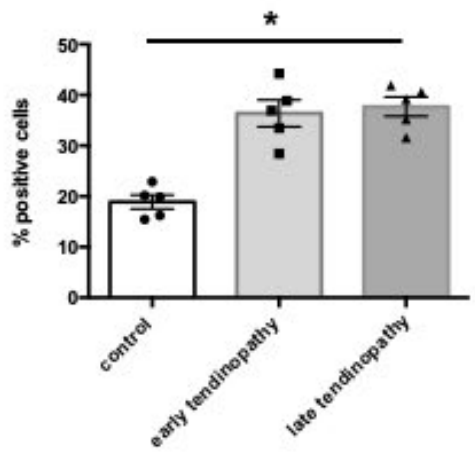

(G)

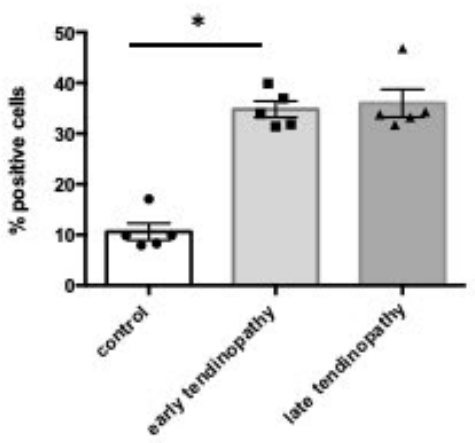

(I)

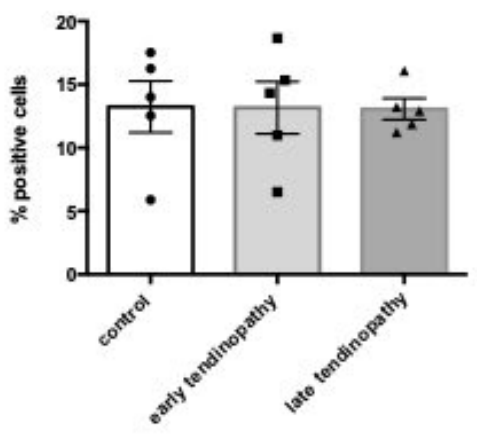



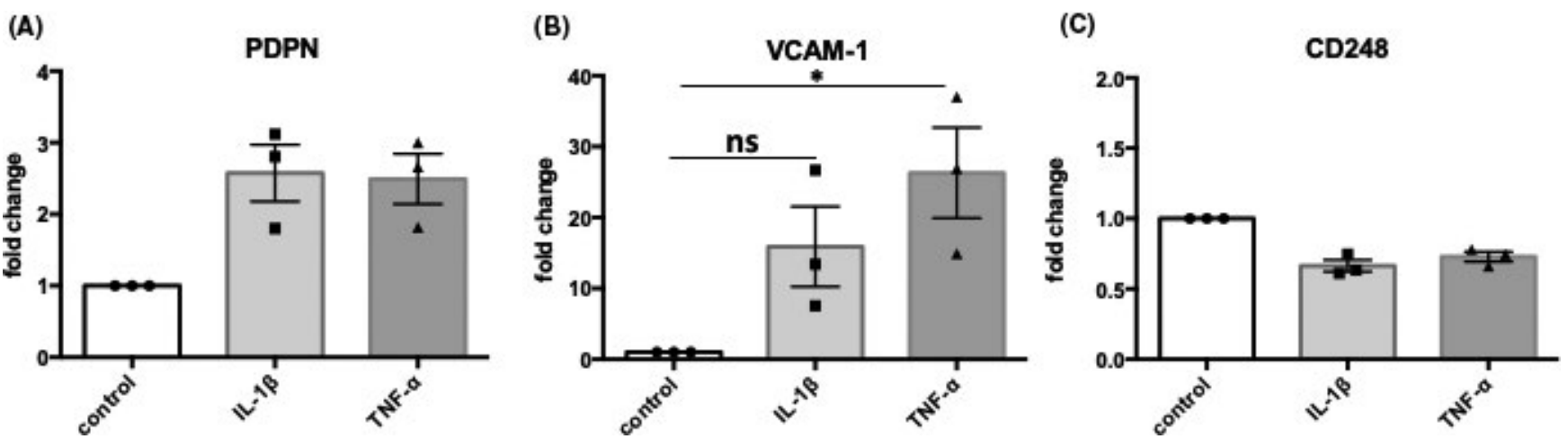

F I G U RE 2 PDPN, VCAM-1, and CD248 expression in tenocytes stimulated with IL-1 $\beta$ and TNF-a. Fold change in mRNA expression (2 ${ }^{-\Delta}$ $\triangle \mathrm{CT}$ ) of (A) PDPN (B) VCAM-1 (C) CD248 expression in normal tenocytes. Data represent mean \pm SEM relative to housekeeping gene GAPDH (mean of duplicate analysis). $* P<.05$ (Friedman test with Dunn's multiple comparisons)
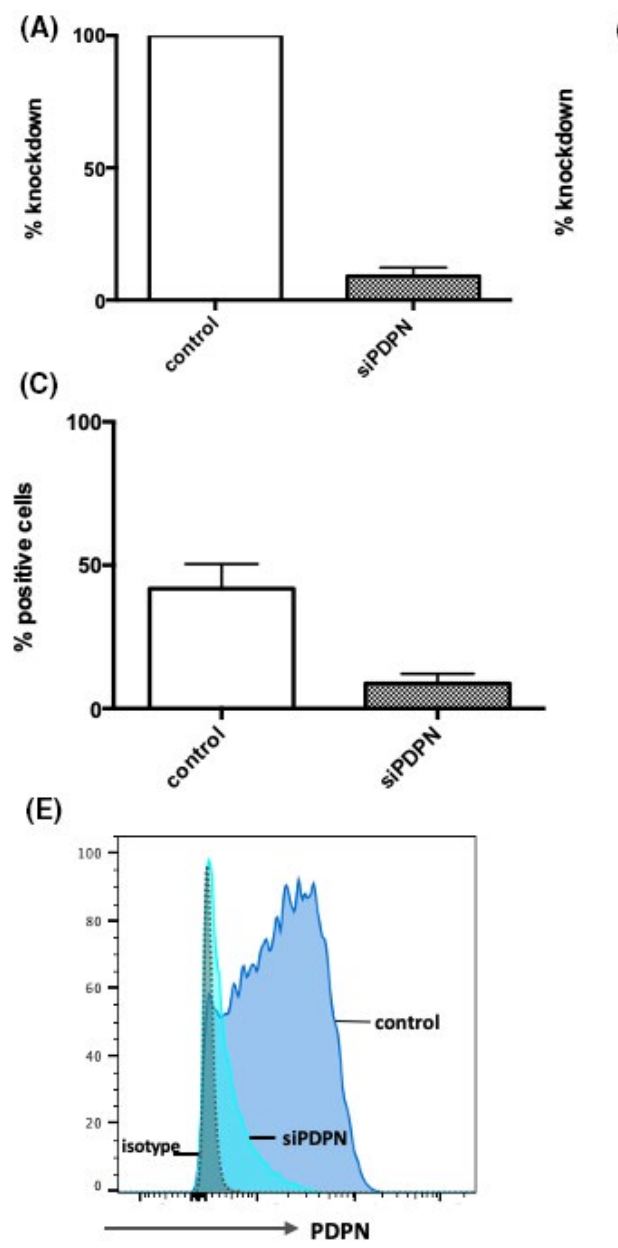
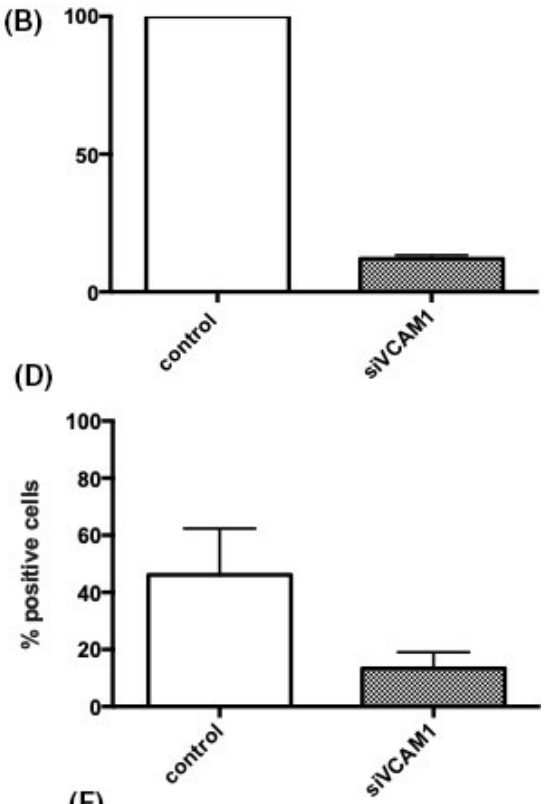

(F)

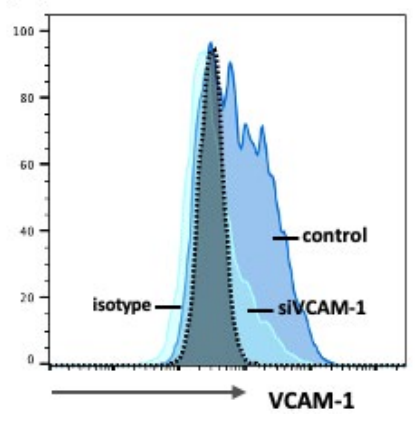

F I G URE 3 Podoplanin and VCAM-1 knockdown in tenocytes. siRNA knockdown of podoplanin in scramble control and transfected tenocytes (siPDPN/siVCAM-1) (A \& B) expressed as \% positive singlet cells based on isotype control gating, $\mathrm{n}=3(\mathrm{C} \& \mathrm{D})$ percentage knockdown of podoplanin measured by RT-PCR (relative to $18 \mathrm{~S}$ endogenous control), $\mathrm{n}=3$ ( $\mathrm{E} \&$ F) representative histogram of podoplanin surface expression in transfected tenocytes relative to scramble control. All data represent mean \pm SEM, $\mathrm{n}=3$ [Colour figure can be viewed at wileyonlinelibrary. com]

F I G U R E 1 PDPN, VCAM-1, and CD248 expression in tendinopathy. Relative mRNA expression ( $\left.2^{\wedge}-\Delta C T\right)$ of (A) PDPN (B) VCAM-1 (C) CD248 expression in control (hamstring tendon, $\mathrm{n}=9$ ), early tendinopathy (intact subscapularis biopsy, $\mathrm{n}=10$ ) and late tendinopathy (torn supraspinatus tendon, $\mathrm{n}=14$ ). Data represent mean \pm SEM relative to housekeeping gene GAPDH (mean of duplicate analysis). $* P<.05$, $* * P<.01$, (Kruskal-Wallis test) vs control. Control and late tendinopathy sections stained with antibodies directed against PDPN (D) VCAM-1 (F) CD248 (H). Quantitative expression of (E) PDPN (G) VCAM-1 (I) CD248 depicts mean percentage of positive cells per sample based on 5 highpower fields, Data represent mean \pm SEM, $\mathrm{n}=5$ for control tendon, early and late tendinopathy. $* P<.05$ (Friedman test with Dunn's multiple comparisons) [Colour figure can be viewed at wileyonlinelibrary.com] 
Loss of podoplanin and VCAM-1 expression did not appear to have any bearing on the cytokine/chemokine profile of tenocytes (measured by expression of IL-6, IL-8, and CCL2) at rest nor did it direct any apparent changes in matrix regulation. From our results, it is evident that IL- $1 \beta$ induces expression of podoplanin and VCAM- 1 in tenocytes that is accompanied by significant production of inflammatory mediators and matrix proteins reflecting the molecular changes observed in tendinopathy. Interestingly, knockdown of podoplanin and VCAM-1 did not affect the responsiveness of tenocytes to an inflammatory challenge indicating there is no direct relationship between expression of stromal "activation" markers and regulation of inflammation and matrix turnover. For this relationship to be deemed directly functional, we would have expected to see a correlation between cytokine/chemokine production and loss of podoplanin/VCAM-1 expression. However, tenocytes lacking podoplanin/VCAM-1 expression appeared to remain equally as responsive as control cells upon stimulation indicating they have retained their inflammatory properties.

Studies in the context of wound healing and fibroproliferative disease have outlined a clear relationship between function and phenotype whereby fibroblast "activation" (characterized by expression of $\alpha$-SMA) is accompanied by excessive pathological matrix production. ${ }^{27,28}$ By contrast, our loss-of-function studies did not identify any such tangible relationship between expression of surface proteins and inflammation/matrix regulation by tenocytes. The first observation of "activation" of fibroblasts and their participation in the innate immune response was described in the rheumatoid synovium whereby engagement of TLR ligands on the cell surface resulted in the release of chemokines. ${ }^{29}$ As such, we propose that the "activation" state of tenocytes
Normal

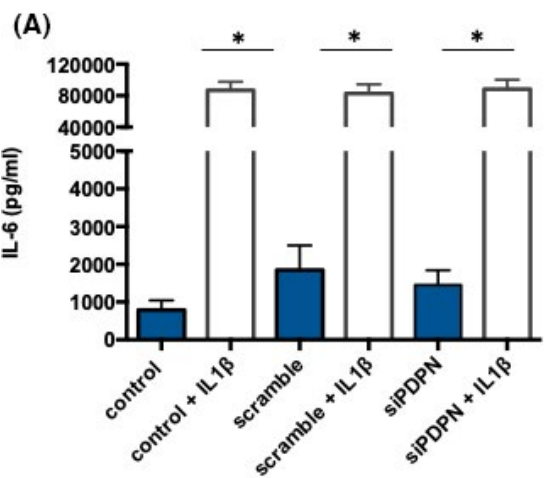

(C)

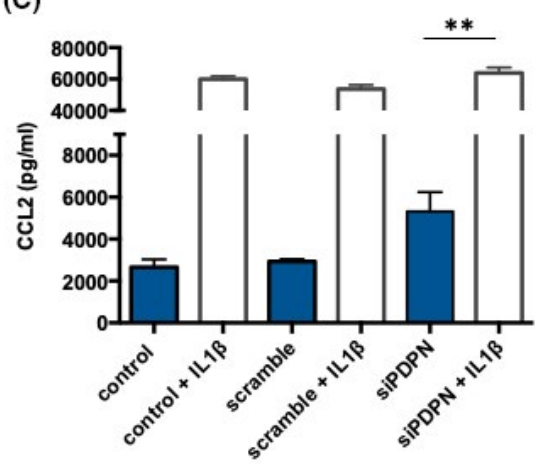

(E)

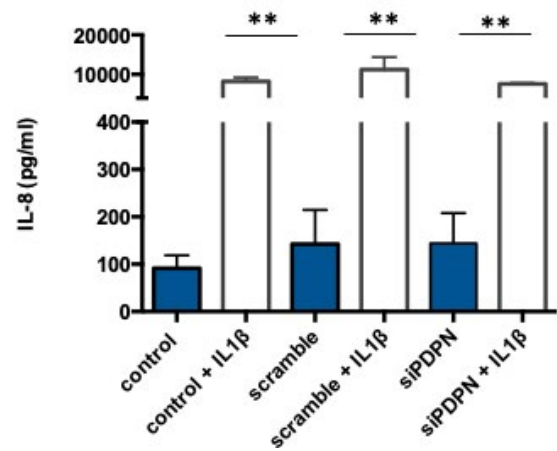

Tendinopathic

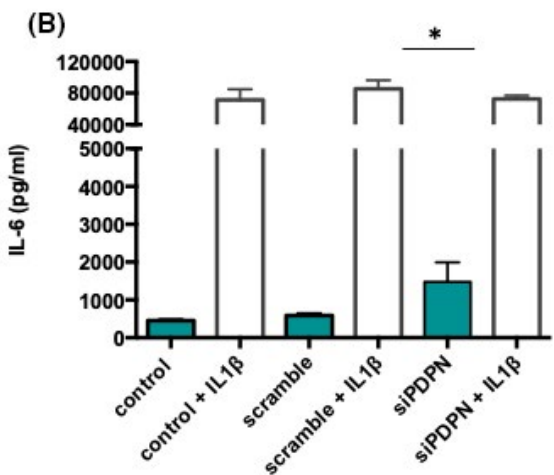

(D)

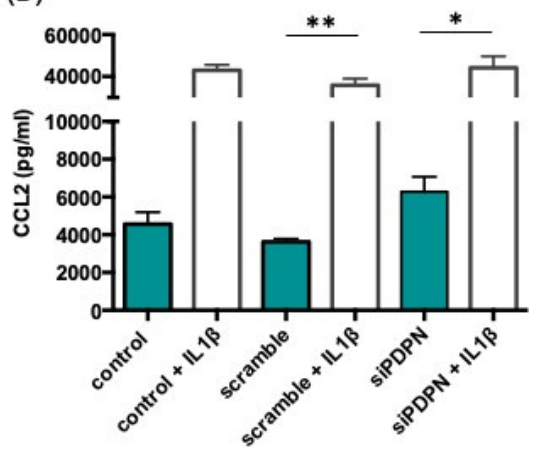

(F)

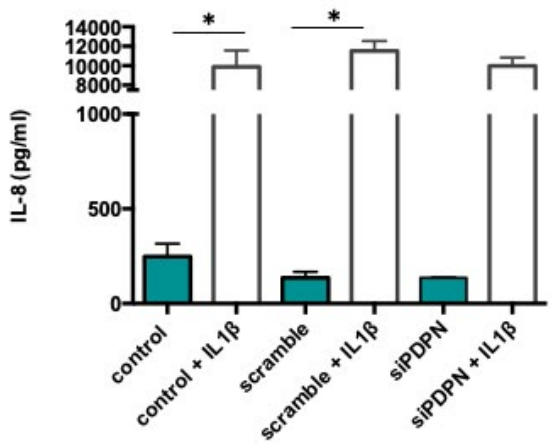



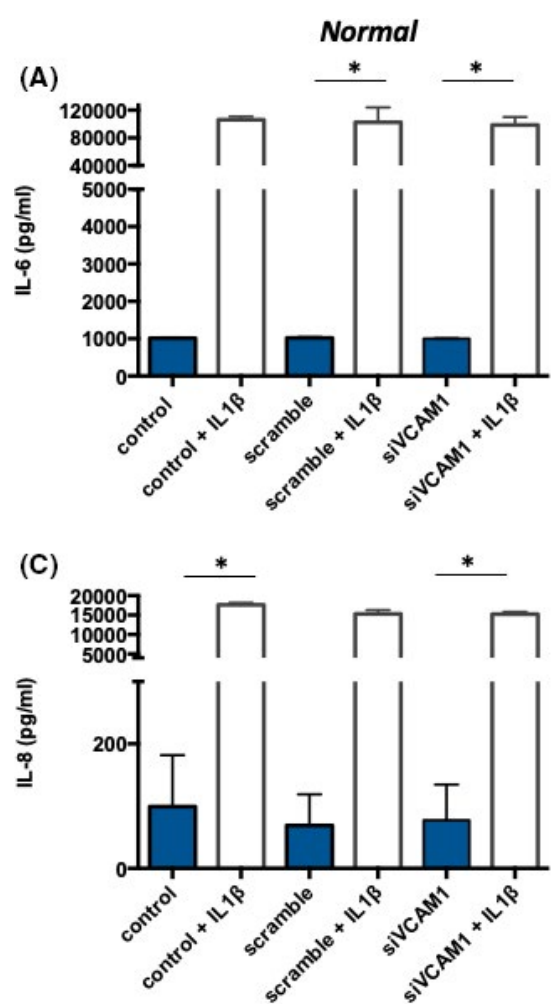

(E)

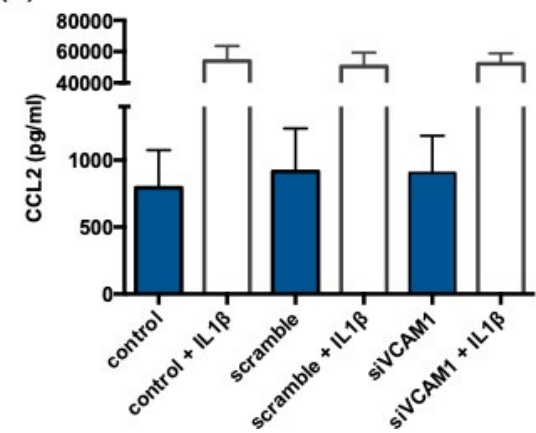

Tendinopathic

(B)

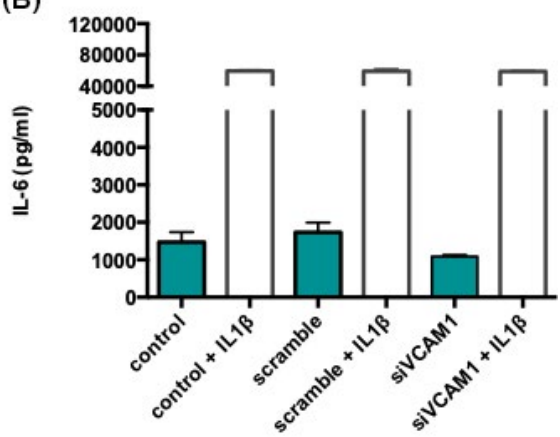

(D)

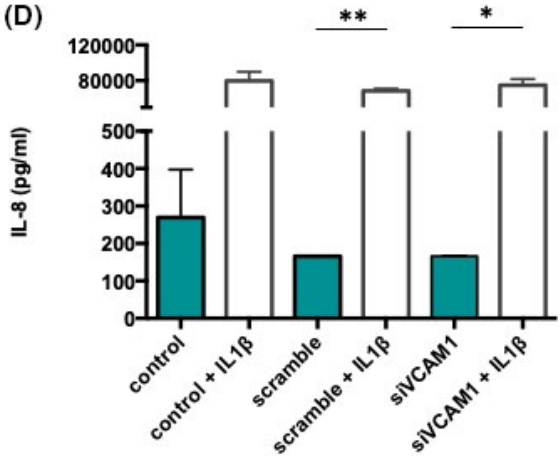

(F)

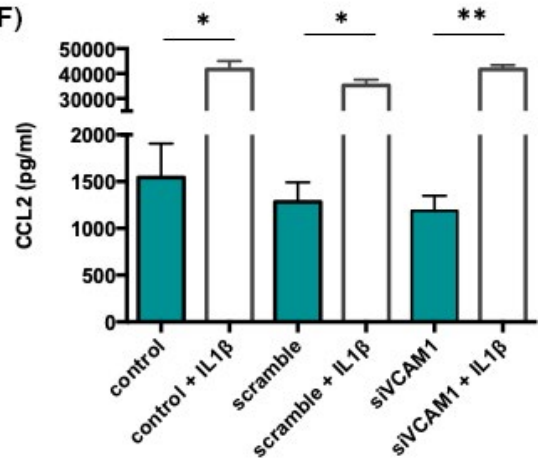

F I G URE 5 Cytokine release from tenocytes with VCAM-1 knockdown. IL-6, IL-8, and CCL2 release from (A, C, E) normal tenocytes (B, D, F) tendinopathic tenocytes left untransfected (control), transfected with scramble control and transfected with podoplanin siRNA in the presence or absence of $10 \mathrm{ng} / \mathrm{mL}$ IL-1 $\beta$ stimulation. $P<.05, * * P<.01$ (One-way ANOVA with Tukey's multiple comparisons test). All data represent mean \pm SEM, $\mathrm{n}=3[$ Colour figure can be viewed at wileyonlinelibrary.com] is more appropriately measured by functional properties including relative expression of inflammatory mediators and matrix proteins rather than potentially arbitrary phenotypic properties.

As podoplanin and VCAM-1 are predominantly expressed on the cell surface we acknowledge, there are limitations to closed system in vitro models that exclude potential receptor-ligand interactions. CLEC-2 is the only known ligand for podoplanin and is expressed at low levels on DCs and macrophages. ${ }^{30}$ It is plausible that engagement of the podoplanin/CLEC-2 axis at the tenocyte-immune cell interface is required to activate signaling cascades that regulate the immune response. ${ }^{21}$ Similarly, VCAM-1 is associated with leukocyte trafficking during inflammation through interaction with $\alpha 4 \beta 1$ integrin. ${ }^{31}$ As such, it is possible that increased and persistent expression podoplanin and VCAM-1 on the tenocyte surface in disease is a priming mechanism that allows stromal cells to engage with infiltrating cells more readily. This may be a result of epigenetic imprinting based on previous trauma or immediate microenvironmental cues present in the tendon stroma. ${ }^{11}$

Recent transcriptomic studies have described the presence of several "subpopulations" of fibroblasts in RA. Anatomical localization of fibroblast subpopulations has been identified through the sublining and lining later of the synovium based on surface marker expression. ${ }^{32-34}$ Gene expression analysis and in vitro validation have also revealed distinct inflammatory profiles of subsets defined by differential cytokine/chemokine expression, enhanced leukocyte recruitment and activation of signaling pathways $^{32,35}$ We postulate that subpopulations of tenocytes with varying surface marker profiles, not limited to those described herein, exist within the tendon stroma. Such populations may possess varying immunological properties including cytokine/chemokine repertoire and regulation of ECM turnover. The concepts of fibroblast "activation" and heterogeneity are inextricably linked and it is possible they represent a "cause and effect" phenomenon whereby 
F I G URE 6 Podoplanin knockdown does not directly alter matrix regulation by tenocytes. (A) COL1A1(B) COL3A1 (C) $T N C$ (D) $D C N$ expression in normal or tendinopathic tenocytes transfected with scramble control or VCAM- 1 siRNA in the presence or absence of $10 \mathrm{ng} / \mathrm{mL} \mathrm{IL-} 1 \beta$ stimulation. All data are normalized to $18 \mathrm{~S}$ housekeeping gene and expressed as fold change relative to scramble control, data represent mean $\pm \mathrm{SEM}, \mathrm{n}=3$ [Colour figure can be viewed at wileyonlinelibrary. com]
COL1A1

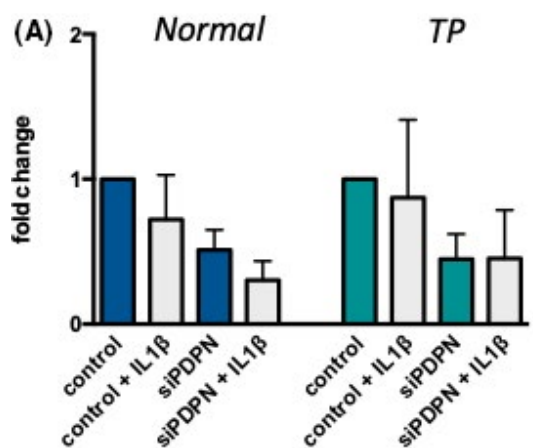

$\underline{T N C}$

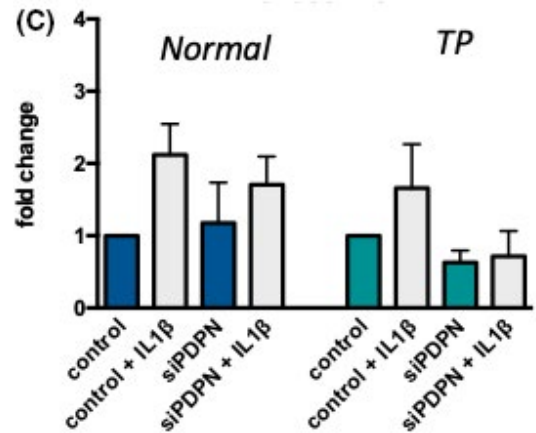

COLIA1

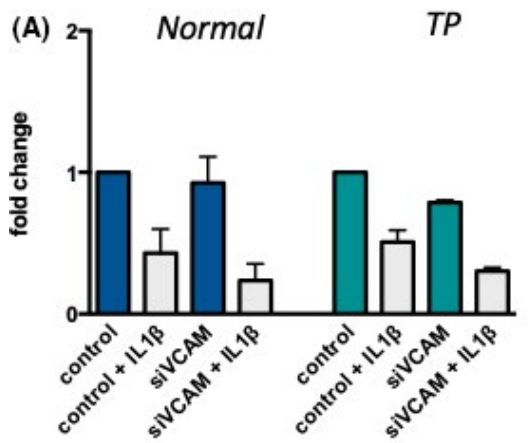

$\underline{T N C}$

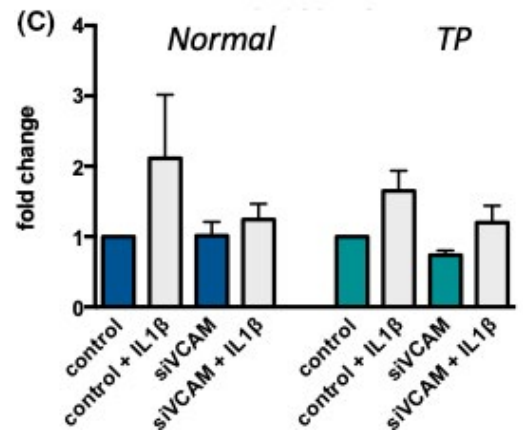

COL3A1

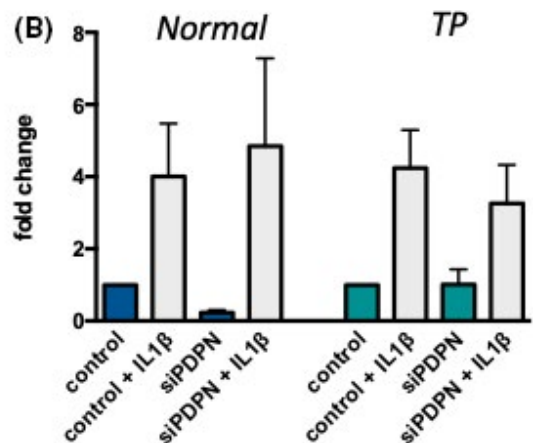

DCN

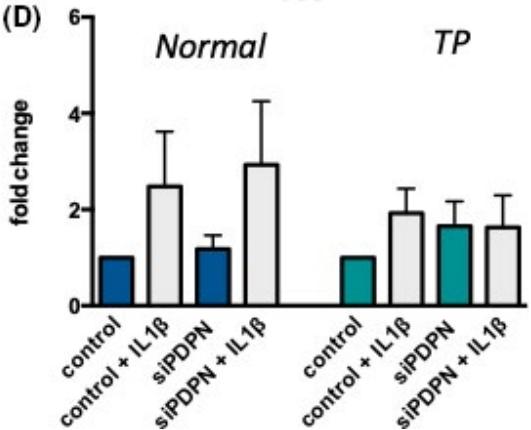

does not directly alter matrix regulation by tenocytes. (A) COL1A1 (B) COL3A1 (C) $T N C$ (D) $D C N$ expression in normal or tendinopathic tenocytes transfected with scramble control or VCAM-1 siRNA in the presence or absence of $10 \mathrm{ng} / \mathrm{mL} \mathrm{IL-} 1 \beta$ stimulation. All data are normalized to 18S housekeeping gene and expressed as fold change relative to scramble control, data represent mean $\pm \mathrm{SEM}, \mathrm{n}=3$ [Colour figure can be viewed at wileyonlinelibrary.

com]

fibroblasts exposed to inflammatory stimuli in the early stages of disease give rise to distinct subpopulations present in established pathology.

While the translation of concepts between disease states that display similar characteristics is invaluable to advancement of knowledge, caution should be exercised with regards to universal terminology such as stromal "activation." When considering stromal biology, drawing comparisons between markers expressed on tenocytes in tendinopathic tendon and RA synovium (RA-FLS) or the 
tumor microenvironment (cancer-associated fibroblasts) may be problematic as each disease state displays a unique immune landscape. ${ }^{17,35}$ For example, in chronic tendon disorders the magnitude of immune cell infiltration to the damaged tissue is far less marked than that of an persistently inflamed joint and different immune cell populations predominate through stages of disease progression. ${ }^{3}$ It is evident that chronic exposure to inflammatory conditions has a lasting effect on the properties stromal cells; however, such properties are likely to be highly tissue specific and dependent upon local microenvironmental cues.

\section{5 | CONCLUSIONS}

The aim of this study was to assess the effect of dampening "activation" of tendon stromal cells through knockdown of surface proteins podoplanin and VCAM-1. We conclude that tenocytes lacking expression of "activation" markers podoplanin and VCAM-1 do not display any discernible changes in typical disease-associated behaviors and remain pathogenic. "Activated" stromal cells are said to be primed to respond to inflammation through stromal memory; however, we found neither the relative presence nor absence of podoplanin/VCAM-1 had any effect on the tenocyte's response to a subsequent inflammatory challenge. Taken together, it is evident that expression of these surface proteins does not directly affect the intrinsic workings of the cell with regard to synthesis and release of inflammatory mediators and matrix proteins that is typical under conditions of tissue stress. We propose that, in the context of tendinopathy, the term "activation" is more appropriately associated with alterations in tenocyte behavior that induce changes in the stromal microenvironment and overall tissue architecture. Identifying mechanisms through which stromal cells contribute to perpetuation of active disease may aid in attenuating pathological inflammation in tendinopathy through pharmacologic intervention.

\section{CONFLICT OF INTEREST}

The authors declare no competing interests.

\section{AUTHOR CONTRIBUTIONS}

LANC, M.A and NLM conceived and designed the experiments. LANC performed experiments. IBM and NLM provided expert advice. All authors analyzed the data. LANC and NLM wrote the paper.

\section{ETHICAL APPROVAL}

All procedures and protocols were approved by the Ethics Committee under the approval number West of Scotland REC (REC14/WS/1035) with informed consent obtained and carried out in accordance with standard operative procedures.

\section{DATA AVAILABILITY STATEMENT}

LANC has access to all the data, and data are available upon request.

\section{ORCID}

Neal L. Millar (D) https://orcid.org/0000-0001-9251-9907

\section{REFERENCES}

1. Kaux J-F, Forthomme B, Goff CL, Crielaard J-M, Croisier J-L. Current opinions on tendinopathy. J Sports Sci Med. 2011;10:238-253.

2. Khan KM, Maffulli N. Tendinopathy: an Achilles' heel for athletes and clinicians. Clin J Sport Med. 1998;8:151-154

3. Millar NL, Murrell GAC, Mcinnes IB. Inflammatory mechanisms in tendinopathy - towards translation. Nat Rev Rheumatol. 2017;13(2):110-122.

4. Sharma P, Maffulli N. Tendon injury and tendinopathy: Healing and repair. J Bone Joint Surg. 2005;87:187-202.

5. Riley G. Tendinopathy-from basic science to treatment. Nat Clin Pract Rheumatol. 2008;4:82-89.

6. Frantz C, Stewart KM, Weaver VM. The extracellular matrix at a glance. J Cell Sci. 2010;123:4195-4200.

7. Wang JHC. Mechanobiology of tendon. $J$ Biomech. 2006:39:1563-1582.

8. Patel R, Filer A, Barone F, Buckley CD. Stroma: fertile soil for inflammation. Best Pract Res Clin. Rheumatol. 2014;28:565-576.

9. Parsonage G, Filer AD, Haworth $\mathrm{O}$, et al. A stromal address code defined by fibroblasts. Trends Immunol. 2005;26:150-156.

10. Akbar M, Gilchrist DS, Kitson SM et al. Targeting danger molecules in tendinopathy: The HMGB1/TLR4 axis. RMD Open. 2017;3:e00456

11. Karouzakis E, Gay RE, Gay S, Neidhart M. Epigenetic control in rheumatoid arthritis synovial fibroblasts. Nat Rev Rheumatol. 2009;5:266-272.

12. Albrengues J, Bertero T, Grasset E, et al. Epigenetic switch drives the conversion of fibroblasts into proinvasive cancer-associated fibroblasts. Nat. Commun. 2015;6.

13. Dakin SG, Coles M, Sherlock JP, Powrie F, Carr AJ, Buckley CD. Pathogenic stromal cells as therapeutic targets in joint inflammation. Nat Rev Rheumatol. 2018;14:714-726.

14. Slany A, Meshcheryakova A, Beer A, Ankersmit H, Paulitschke V, Gerner C. Plasticity of fibroblasts demonstrated by tissue-specific and function-related proteome profiling. Clin Proteomics. 2014;11(1):41.

15. Ospelt C. Synovial fibroblasts in 2017. RMD Open. 2017;3(2):e000471.

16. Multhoff G, Molls M, Radons J. Chronic inflammation in cancer development. Front Immunol. 2012;2.

17. Sugimoto H, Mundel TM, Kieran MW, Kalluri R. Identification of fibroblast heterogeneity in the tumor microenvironment. Cancer Biol Ther. 2006;5:1640-1646.

18. Akbar M, McLean M, Garcia-Melchor E, et al. Fibroblast activation and inflammation in frozen shoulder. PLoS One. 2019;14:e0215301. 
19. Dakin SG, Buckley CD, Al-Mossawi MH, et al. Persistent stromal fibroblast activation is present in chronic tendinopathy. Arthritis Res Ther. 2017;19:16.

20. Astarita JL, Acton SE, Turley SJ. Podoplanin: Emerging functions in development, the immune system, and cancer. Front Immunol. 2012;3:283.

21. Rayes J, Lax S, Wichaiyo S, et al. The podoplanin-CLEC-2 axis inhibits inflammation in sepsis. Nat Commun. 2017;8.

22. Kong DH, Kim YK, Kim MR, Jang JH, Lee S. Emerging roles of vascular cell adhesion molecule-1 (VCAM-1) in immunological disorders and cancer. Int J Mol Sci. 2018;19:1057.

23. Cher JZBZB, et al. Alarmins in frozen shoulder: a molecular association between inflammation and pain. Am J Sport Med. 2018;46(3):671-678.

24. Valdez Y, Maia M, Conway ME. CD248: Reviewing its Role in Health and Disease. Curr Drug Targets. 2012;13:432-439.

25. Hardie DL, Baldwin MJ, Naylor A, et al. The stromal cell antigen CD248 (endosialin) is expressed on naive CD8+ human T cells and regulates proliferation. Immunology. 2011;133:288-295.

26. Rettig WJ, Garin-Chesa P, Healey JH, et al. Regulation and heteromeric structure of the fibroblast activation protein in normal and transformed cells of mesenchymal and neuroectodermal origin. Cancer Res. 1993;53:3327-3335.

27. Rognoni E, Watt FM. Skin cell heterogeneity in development, wound healing, and cancer. Trends Cell Biol. 2018;28:709-722.

28. Wynn TA, Ramalingam TR. Mechanisms of fibrosis: therapeutic translation for fibrotic disease. Nat Med. 2012;18:1028-1040.

29. Smith RS, Smith TJ, Blieden TM, Phipps RP. Fibroblasts as sentinel cells. Synthesis of chemokines and regulation of inflammation. Am J Pathol. 1997;151:317-322.

30. Colonna M, Samaridis J, Angman L. Molecular characterization of two novel C-type lectin-like receptors, one of which is selectively expressed in human dendritic cells. Eur J Immunol. 2000;30:697-704.

31. Cook-Mills JM, Marchese ME, Abdala-Valencia H. Vascular cell adhesion molecule-1 expression and signaling during disease: Regulation by reactive oxygen species and antioxidants. Antioxid Redox Signal. 2011;15:1607-1638.

32. Mizoguchi F, Slowikowski K, Wei K, et al. Functionally distinct disease-associated fibroblast subsets in rheumatoid arthritis. Nat Commun. 2018;9.

33. Stephenson W, et al. Single-cell RNA-seq of rheumatoid arthritis synovial tissue using low-cost microfluidic instrumentation. Nat. Commun. 2018;9.

34. Croft AP, Campos J, Jansen K, et al. Distinct fibroblast subsets drive inflammation and damage in arthritis. Nature. 2019;570:246-251.

35. Zhang F, Wei K, Slowikowski K, et al. Defining inflammatory cell states in rheumatoid arthritis joint synovial tissues by integrating single-cell transcriptomics and mass cytometry. Nat Immunol. 2019;20(7):928-942.

\section{SUPPORTING INFORMATION}

Additional supporting information may be found online in the Supporting Information section.

How to cite this article: Crowe LAN, Garcia Melchor E, Murrell GAC, McInnes IB, Akbar M, Millar NL. Stromal "activation" markers do not confer pathogenic activity in tendinopathy. Transl Sports Med.

2021;4:268-279. https://doi.org/10.1002/tsm2.204 